\title{
Biogeography of freshwater fishes from the Northeastern Mata Atlântica freshwater ecoregion: distribution, endemism, and area relationships
}

\author{
Priscila Camelier ${ }^{1}$ and Angela M. Zanata ${ }^{2}$
}

The Northeastern Mata Atlântica freshwater ecoregion (NMAF) includes part of the eastern Brazilian coastal drainages, has high level of fish endemism and great biogeographic significance. A taxonomic inventory of freshwater fishes from 25 drainages of the NMAF ecoregion and a biogeographic analysis using the Parsimony Analysis of Endemicity (PAE) method were carried out. A total of 192 native species was listed. The PAE method was applied to 24 basins and 37 species, resulting in five equally parsimonious area diagrams. The strict consensus diagram indicates the existence of two main groups of basins throughout the NMAF ecoregion. These groups were denominated: North Group and Central-South Group. The CentralSouth Group shows a basal polytomy composed by two Groups (Central Group and South Group) plus the rio Itapemirim basin. The North Group is composed by eight drainages from the rio Sergipe to the rio Paraguaçu, the Central Group by five drainages from the rio Cachoeira to the rio Jequitinhonha, and the South Group by nine drainages from the rio Buranhém to the rio Doce. Comments about the species distribution and the fish fauna shared with adjacent ecoregions are provided. We also present a comparison of the hypothesis of river relationships proposed herein with published phylogenetic hypotheses that include taxa relevant to this study.

A ecorregião Mata Atlântica Nordeste (NMAF) inclui parte das drenagens costeiras do leste do Brasil, tem alto grau de endemismo de peixes e grande significado biogeográfico. Um levantamento taxonômico da ictiofauna de água doce de 25 drenagens da ecorregião NMAF e uma análise biogeográfica utilizando o método de Análise de Parcimônia de Endemismo (PAE) foram realizados. Um total de 192 espécies nativas foi listado. O método PAE foi aplicado a 24 bacias e 37 espécies, resultando em cinco diagramas de áreas igualmente parcimoniosos. O diagrama de consenso estrito indica a existência de dois grupos de bacias principais ao longo da ecorregião NMAF. Estes grupos foram denominados: Grupo Norte e Grupo Centro-Sul. O Grupo Centro-Sul apresenta uma politomia basal composta por dois grupos (Grupo Centro e Grupo Sul) mais a bacia do rio Itapemirim. O Grupo Norte é formado por oito drenagens entre o rio Sergipe e o rio Paraguaçu, o Grupo Centro por cinco drenagens entre o rio Cachoeira e o rio Jequitinhonha e o Grupo Sul por nove drenagens entre o rio Buranhém e rio Doce. Comentários sobre a distribuição das espécies e a fauna compartilhada com ecorregiões adjacentes são fornecidos. Apresentamos também uma comparação da hipótese de relação aqui obtida com filogenias publicadas para alguns táxons relevantes ao presente estudo.

Key words: Coastal drainages, Eastern Brazil, Neotropical region, PAE, River relationships.

\section{Introduction}

Although freshwater fishes offer interesting opportunities to formulate biogeographical hypotheses, the biogeography of Neotropical ichthyofauna is poorly understood. According to Vari \& Weitzman (1990), there are three major factors that limit the precision of hypotheses about the historical biogeography of South American freshwater fishes: limited knowledge of species-level taxonomy, inadequate distributional information for most species, and sparse or non-existent data on the phylogenetic history of most supraspecific taxa. These factors are still affecting the knowledge of the Neotropical freshwater fish biogeography, although considerable advances were achieved in the last 25 years. Recently, Ribeiro (2006) added a fourth factor related to the difficulties in associating phylogenetic hypotheses with the known geological history of the continent. When available, geological inferences refer to Andean and lowland basin evolution, while little information is provided about the geological history of the eastern portion of the Brazilian shield (e.g., Lundberg et al., 1998; Buckup, 2011; Lima \& Ribeiro, 2011). Fortunately, a summary of the tectonic events that dominated the geological

${ }^{1}$ Museu de Zoologia da Universidade de São Paulo, Caixa Postal 42494, 04218-970 São Paulo, SP, Brazil. pricamelier@gmail.com (corresponding author)

${ }^{2}$ Departamento de Zoologia, Instituto de Biologia, Universidade Federal da Bahia, Câmpus de Ondina, Rua Barão de Geremoabo s/n, 40170-290 Salvador, BA, Brazil. zanata.angela@gmail.com 
evolution of the coastal drainages of eastern Brazil was provided by Ribeiro (2006), who proposed distributional patterns of fish groups that correspond to general patterns of relationships between the inland and coastal drainages associated with the eastern limits of the Brazilian shield. These biogeographic patterns, based on deep phylogenetic lineages as well as closely related species groups occurring in coastal drainages and the adjacent upland crystalline shield rivers, reinforce the historical connections of some of the interior and coastal drainages. In fact, the contribution of the hydrographic basins from the continental interior to the process of ichthyofaunistic formation of eastern Brazilian rivers was previously acknowledged by various authors (e.g., Menezes, 1972; Bizerril, 1994; Costa, 1996, 2001, 2003), indicating the existence of an ichthyofauna of mixed origin (Rosa et al., 2004). More recently, Buckup (2011) reviewed historical biogeographic evidence associated with general distribution patterns of the fish fauna occurring on the eastern limits of the Brazilian Shield but, in that case, there was a greater emphasis on the coastal fauna of southeastern Brazil and the boundaries of the upper rio Paraná and rio São Francisco basins.

Some of the main Atlantic coastal drainages include, from north to south, the Paraguaçu, rio de Contas, Pardo, and Doce basins, which have a relatively broadly branched system of headwaters that abut the eastern headwaters of the rio São Francisco basin, but are separated from them by the highlands of the Espinhaço complex (Buckup, 2011). The geological evolution of the high chain mountains associated with the Espinhaço complex provided an opportunity for vicariance and isolation of the fish in the independent coastal rivers. Thus, apart from the sister group relationships and shared species between coastal drainages and interior continental basins, the coastal drainages of eastern Brazil have been recognized as a very distinct area in terms of their ichthyofauna, mainly expressed by its high endemism at the species and genus levels. According to Bizerril (1994), of the 285 fish species present in the coastal basins, $95 \%$ were found to be endemic, with $23.4 \%$ of the genera endemic. However, the coastal basins draining the eastern edge of the Brazilian shield do not comprise a uniform biogeographic area of endemism.

Bizerril (1994) recognized two subprovinces in the eastern region, the southeast coast and the east coast, based on the presence or absence of certain genera. The southeastern subprovince defined by Bizerril (1994) was recently studied by Carvalho (2007), who divided the region into four groups of drainages based on parsimony analysis of endemism. No further detailed analysis was performed for the eastern subprovince as defined by Bizerril (1994). Thus, almost no biogeographic studies include the northeastern coastal basins, and, in general, the few studies available are restricted to a particular taxonomic group with known occurrence in one or two drainages of the east Brazilian coast (e.g., Vari, 1988; Schaefer, 1997; Costa, 2001, 2003; Menezes et al., 2008). Furthermore, most of these studies focus only on the description of the geographic distribution of species, and just a few of them make use of biogeographical methods (e.g., Costa, 2003; Montoya-Burgos, 2003; Sarmento-Soares et al., 2009a).

The "subprovince of the east coast" sensu Bizerril (1994) is bordered by the rio Paraíba do Sul basin to the south and by the coastal basins in Bahia south of the rio São Francisco outlet. This subprovince is almost equivalent to "Northeastern Mata Atlântica freshwater ecoregion" (ecoregion 328) proposed by Abell et al. (2008). According to these authors, a freshwater ecoregion is defined as a large area encompassing one or more freshwater systems with a distinct assemblage of natural freshwater communities and species. Thus, it is expected that the freshwater species and environmental conditions within a given ecoregion are more similar to each other than to those of surrounding ecoregions. The Northeastern Mata Atlântica freshwater ecoregion (NMAF ecoregion) includes all coastal drainages in eastern Brazil in the area from the rio Sergipe in the north to the rio Itabapoana in the south. To the west the NMAF ecoregion is limited by the drainage divide with the rio São Francisco basin along the Serra do Espinhaço (Hales \& Petry, 2013).

In this study, we propose a general hypothesis about the relationships of the main drainages comprising the NMAF ecoregion. We also provide comments about species distributions and endemism, a comparison of the hypothesis of river relationships proposed herein with published phylogenetic hypotheses that include taxa relevant to this study, and a brief analysis of the geomorphological information available for the drainages of interest.

\section{Material and Methods}

This study included 25 drainages of the NMAF ecoregion, in the area between border of the rio São Francisco basin on the north and rio Paraíba do Sul basin on the south. From north to south, the main drainages of NMAF ecoregion are: Sergipe, Vaza-Barris, Piauí, Real, Itapicuru, Inhambupe, Pojuca, Paraguaçu, Contas, Cachoeira, Almada, Una, Pardo, Jequitinhonha, Buranhém, Frades, Jucuruçu, Itanhém, Peruípe, Mucuri, Itaúnas, São Mateus, Doce, Itapemirim, and Itabapoana (Fig. 1). The species inventory supporting this study was based on sampling conducted at approximately 150 collection sites during expeditions carried out between 2004 and 2013 and in specimens examined previously deposited in the collections of Museu Nacional, Rio de Janeiro (MNRJ), Museu de Zoologia da Universidade de São Paulo, São Paulo (MZUSP), and Museu de Zoologia da Universidade Federal da Bahia, Salvador (UFBA). Voucher specimens gathered on the expeditions were deposited in the collections of UFBA and MZUSP. Information about the distribution of the species utilized in the biogeograpical analysis was obtained from the species confidently identified in the present study and from reliable published literature (i.e., original descriptions, taxonomic reviews, phylogenetic studies, and catalogs of species). Authorships of the species analyzed are available in Table 1. A diagram 
of biogeographical area relationships was produced using parsimony analysis of endemicity (PAE) as proposed by Rosen (1988) and discussed by Rosen \& Smith (1988), Cracraft (1991), and Rosen (1992). In PAE, the distribution of taxa across sampled localities is treated as analogous to character distributions proposed by Hennig (1966). After defining the taxonomic composition of each basin, a binary matrix of species $v$ s. drainages (absence $=0$; presence $=$ 1) was produced using Mesquite version 2.72 (Maddison $\&$ Maddison, 2009). Only species endemic to rivers of the NMAF ecoregion were utilized in the analysis. Thus, species shared with adjacent basins (e.g., São Francisco, Paraíba do Sul) were not included in the PAE analysis, but the sharing with those river basins was discussed. Single basin endemics and species widely distributed were also not included because they are biogeographically uninformative. Exotic or taxonomically complex species were not included in the matrix because they may generate misleading information about basin relationships. The Itabapoana basin was considered undersampled (no or one informative species) and was not included in the PAE analysis. A hypothetical ancestral area in which all species were absent (all 0) was added to the matrix to root the tree (Rosen \& Smith, 1988). The parsimony analysis was carried out using heuristic search algorithms in the program TNT version 1.1 (Goloboff et al., 2008) with 100 replicates, 10,000 randomizations, and 10 trees saved per replicate. The five most parsimonious trees obtained were summarized using strict consensus.

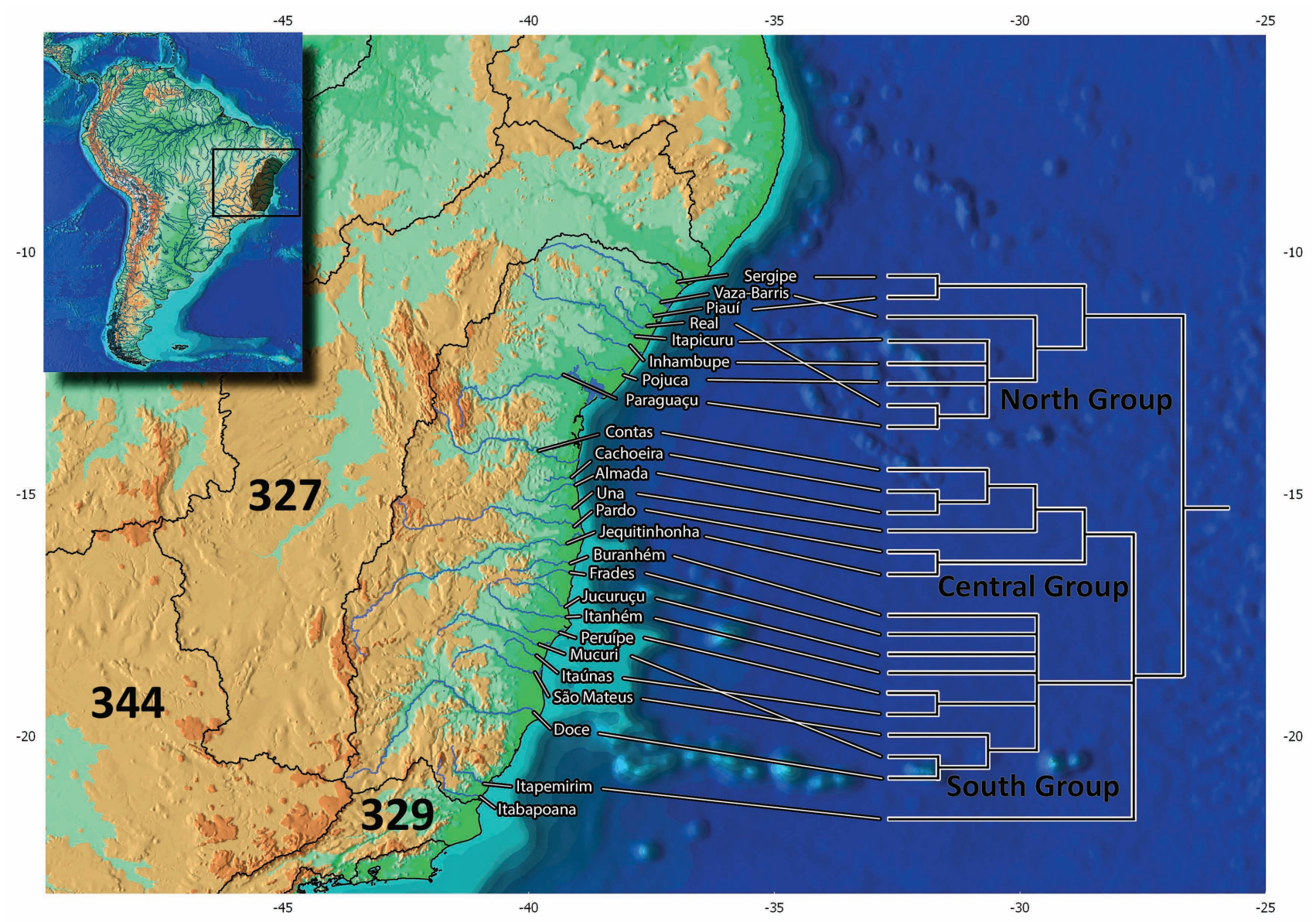

Fig. 1. Map showing the Northeastern Mata Atlântica ecoregion, the rivers included in the PAE, and the groups recovered from the analysis. Adjacent freshwater ecoregions are: (327) São Francisco, (329) Paraíba do Sul, and (344) Upper Paraná.

\section{Results and Discussion}

In total, 192 native species of freshwater fishes were recorded across the 25 drainages surveyed (Table 1). Species of the genus Geophagus Heckel and some species of the genera Astyanax Baird \& Girard (mainly A. bimaculatus group) and Hypostomus Lacepède known to occur in the NMAF ecoregion were not included in the list of species due the various taxonomic problems related to them. Of the 192 species, 155 were excluded from the parsimony analysis as either biogeographically uninformative (i.e., occur in only one drainage $\mathrm{n}=$ 86), potentially misleading (i.e., shared with adjacent ecoregions or widespread in the Neotropical region, $\mathrm{n}=53)$, or taxonomically problematic $(\mathrm{n}=16)$. The remaining 37 species used to build the matrix were securely diagnosed and considered relevant to establish relationships among the 24 drainages (Table 2). 
Table 1. List of species analyzed in this study and its distribution in the Northeastern Mata Atlântica freshwater ecoregion (NMAF) and adjacent ecoregions. $\mathrm{NG}=$ North Group, $\mathrm{CG}=$ Central Group, and $\mathrm{SG}=$ South Group. Species in bold were used to biogeographical analysis. Species occurrences are indicated by an $\mathrm{X}$.

\begin{tabular}{|c|c|c|c|c|c|c|}
\hline \multirow{3}{*}{ SPECIES } & \multicolumn{6}{|c|}{ ECOREGIONS } \\
\hline & \multicolumn{3}{|c|}{ NMAF } & \multirow{2}{*}{ São Francisco } & \multirow{2}{*}{ Paraíba do Sul } & \multirow{2}{*}{ Upper Paraná } \\
\hline & NG & $\mathrm{CG}$ & SG & & & \\
\hline CHARACIFORMES & & & & & & \\
\hline Apareiodon itapicuruensis Eigenmann \& Henn, 1916 & $\mathrm{X}$ & $\mathrm{X}$ & & & & \\
\hline Curimatella lepidura (Eigenmann \& Eigenmann, 1889) & $\mathrm{X}$ & & & $\mathrm{X}$ & & \\
\hline Cyphocharax gilbert (Quoy \& Gaimard, 1824) & $\mathrm{X}$ & $\mathrm{X}$ & $\mathrm{X}$ & $\mathrm{X}$ & $\mathrm{X}$ & \\
\hline Cyphocharax pinnilepis Vari, Zanata \& Camelier, 2010 & & $\mathrm{X}$ & & & & \\
\hline Steindachnerina elegans (Steindachner, 1875) & $\mathrm{X}$ & $\mathrm{X}$ & & $\mathrm{X}$ & & \\
\hline Prochilodus brevis Steindachner, 1875 & $\mathrm{X}$ & $\mathrm{X}$ & & $\mathrm{X}$ & & \\
\hline Prochilodus costatus Valenciennes, 1850 & $\mathrm{X}$ & & & $\mathrm{X}$ & & \\
\hline Prochilodus hartii Steindachner, 1875 & & $\mathrm{X}$ & & & & \\
\hline Prochilodus vimboides Kner, 1859 & & & $\mathrm{X}$ & $\mathrm{X}$ & $\mathrm{X}$ & $\mathrm{X}$ \\
\hline Hypomasticus garmani (Borodin, 1929) & & $\mathrm{X}$ & & & & \\
\hline Hypomasticus mormyrops (Steindachner, 1875) & & & $\mathrm{X}$ & & $\mathrm{X}$ & \\
\hline Leporinus bahiensis Steindachner, 1875 & $\mathrm{X}$ & $\mathrm{X}$ & & & & \\
\hline Leporinus brinco Birindelli, Bristki \& Garavello, 2013 & & $\mathrm{X}$ & & & & \\
\hline Leporinus conirostris Steindachner, 1875 & & & $\mathrm{X}$ & & $\mathrm{X}$ & \\
\hline Leporinus copelandii Steindachner, 1875 & & & $\mathrm{X}$ & & $\mathrm{X}$ & \\
\hline Leporinus elongatus Valenciennes, 1850 & & $\mathrm{X}$ & & & & \\
\hline Leporinus piau Fowler, 1941 & $\mathrm{X}$ & & & $\mathrm{X}$ & & \\
\hline Leporinus steindachneri Eigenmann, 1907 & & $\mathrm{X}$ & $\mathrm{X}$ & & $\mathrm{X}$ & \\
\hline Leporinus taeniatus Lütken, 1875 & $\mathrm{X}$ & & & $\mathrm{X}$ & & \\
\hline Leporinus sp. & & $\mathrm{X}$ & & & & \\
\hline Characidium bahiense Almeida, 1971 & $\mathrm{X}$ & & & & & \\
\hline Brycon ferox Steindachner, 1877 & & & $\mathrm{X}$ & & & \\
\hline Brycon vermelha Lima \& Castro, 2000 & & $\mathrm{X}$ & & & & \\
\hline Brycon sp. 1 & & $\mathrm{X}$ & & & & \\
\hline Brycon sp. 2 & & $\mathrm{X}$ & & & & \\
\hline Henochilus wheatlandii Garman, 1890 & & & $\mathrm{X}$ & & & \\
\hline Phenacogaster franciscoensis Eigenmann, 1911 & $\mathrm{X}$ & $\mathrm{X}$ & & $\mathrm{X}$ & & \\
\hline Acinocheirodon melanogramma Malabarba \& Weitzman, 1999 & & $\mathrm{X}$ & & $\mathrm{X}$ & & \\
\hline Compsura heterura Eigenmann, 1915 & $\mathrm{X}$ & & & $\mathrm{X}$ & & \\
\hline Kolpotocheirodon figueiredoi Malabarba, Lima \& Weitzman, 2004 & $\mathrm{X}$ & & & & & \\
\hline Serrapinnus heterodon (Eigenmann, 1915) & $\mathrm{X}$ & $\mathrm{X}$ & & $\mathrm{X}$ & & $\mathrm{X}$ \\
\hline Serrapinnus piaba (Lütken, 1875) & $\mathrm{X}$ & $\mathrm{X}$ & & $\mathrm{X}$ & & $\mathrm{X}$ \\
\hline Serrapinnus sp. 1 & $\mathrm{X}$ & & & & & \\
\hline Serrapinnus sp. 2 & & $\mathrm{X}$ & & & & \\
\hline Lepidocharax burnsi Ferreira, Menezes \& Quagio-Grassiotto, 2011 & & & $\mathrm{X}$ & $\mathrm{X}$ & & \\
\hline Lepidocharax diamantina Ferreira, Menezes \& Quagio-Grassiotto, 2011 & $\mathrm{X}$ & & & & & \\
\hline Mimagoniates microlepis (Steindachner, 1877) & & $\mathrm{X}$ & $\mathrm{X}$ & & $\mathrm{X}$ & $\mathrm{X}$ \\
\hline Mimagoniates sylvicola Menezes \& Weitzman, 1990 & $\mathrm{X}$ & $\mathrm{X}$ & & & & \\
\hline Serrasalmus brandti Lütken, 1875 & $\mathrm{X}$ & & & $\mathrm{X}$ & & \\
\hline Serrasalmus sp. & $\mathrm{X}$ & & & & & \\
\hline Astyanax burgerai Zanata \& Camelier, 2009 & & $\mathrm{X}$ & & & & \\
\hline Astyanax epiagos Zanata \& Camelier, 2008 & $\mathrm{X}$ & & & & & \\
\hline Astyanax hamatilis Camelier \& Zanata, 2014 & $\mathrm{X}$ & & & & & \\
\hline Astyanax jacobinae Zanata \& Camelier, 2008 & $\mathrm{X}$ & & & & & \\
\hline Astyanax microschemos Bertaco \& Lucena, 2006 & & & $\mathrm{X}$ & & & \\
\hline
\end{tabular}




\begin{tabular}{|c|c|c|c|c|c|c|}
\hline \multirow{3}{*}{ SPECIES } & \multicolumn{6}{|c|}{ ECOREGIONS } \\
\hline & \multicolumn{3}{|c|}{ NMAF } & \multirow{2}{*}{ São Francisco } & \multirow{2}{*}{ Paraíba do Sul } & \multirow{2}{*}{ Upper Paraná } \\
\hline & NG & $\mathrm{CG}$ & SG & & & \\
\hline Astyanax pelecus Bertaco \& Lucena, 2006 & & $\mathrm{X}$ & & & & \\
\hline Astyanax turmalinensis Triques, Vono \& Caiafa, 2003 & & $\mathrm{X}$ & & & & \\
\hline Astyanax vermilion Zanata \& Camelier, 2009 & & $\mathrm{X}$ & & & & \\
\hline Astyanax sp. 1 & $\mathrm{X}$ & $\mathrm{X}$ & & & & \\
\hline Astyanax sp. 2 & $\mathrm{X}$ & $\mathrm{X}$ & $\mathrm{X}$ & & & \\
\hline Astyanax sp. 3 & $\mathrm{X}$ & & & & & \\
\hline Astyanax sp. 4 & $\mathrm{X}$ & & & & & \\
\hline Astyanax sp. 5 & $\mathrm{X}$ & & & & & \\
\hline Astyanax sp. 6 & & $\mathrm{X}$ & & & & \\
\hline Astyanax sp. 7 & $\mathrm{X}$ & & & & & \\
\hline Astyanax sp. 8 & & $\mathrm{X}$ & $\mathrm{X}$ & & & \\
\hline Hasemania piatan Zanata \& Serra, 2010 & & $\mathrm{X}$ & & & & \\
\hline Hemigrammus brevis Ellis, 1911 & $\mathrm{X}$ & & & $\mathrm{X}$ & & \\
\hline Hemigrammus marginatus Ellis, 1911 & $\mathrm{X}$ & $\mathrm{X}$ & & $\mathrm{X}$ & & $\mathrm{X}$ \\
\hline Hyphessobrycon bifasciatus Ellis, 1911 & & & $\mathrm{X}$ & & $\mathrm{X}$ & $\mathrm{X}$ \\
\hline Hyphessobrycon brumado Zanata \& Camelier, 2010 & & $\mathrm{X}$ & & & & \\
\hline Hyphessobrycon itaparicensis Lima \& Costa, 2001 & $\mathrm{X}$ & & & & & \\
\hline Hyphessobrycon micropterus (Eigenmann, 1915) & $\mathrm{X}$ & & & $\mathrm{X}$ & & \\
\hline Hyphessobrycon negodagua Lima \& Gerhard, 2001 & $\mathrm{X}$ & & & & & \\
\hline Hyphessobrycon parvellus Ellis, 1911 & $\mathrm{X}$ & & & & & \\
\hline Hyphessobrycon vinaceus Bertaco, Malabarba \& Dergam, 2007 & & $\mathrm{X}$ & & & & \\
\hline Hyphessobrycon sp. 1 & & $\mathrm{X}$ & & & & \\
\hline Hyphessobrycon sp. 2 & $\mathrm{X}$ & & & & & \\
\hline Hyphessobrycon sp. 3 & $\mathrm{X}$ & & & & & \\
\hline Hyphessobrycon sp. 4 & & & $\mathrm{X}$ & & & \\
\hline Moenkhausia costae (Steindachner, 1907) & $\mathrm{X}$ & & & $\mathrm{X}$ & & \\
\hline Moenkhausia diamantina Benine, Castro \& Santos, 2007 & $\mathrm{X}$ & & & & & \\
\hline Moenkhausia doceana (Steindachner, 1877) & & & $\mathrm{X}$ & & & \\
\hline Myxiops aphos Zanata \& Akama, 2004 & $\mathrm{X}$ & & & & & \\
\hline Nematocharax venustus Weitzman, Menezes \& Britski, 1986 & & $\mathrm{X}$ & & & & \\
\hline Oligosarcus acutirostris Menezes, 1987 & & $\mathrm{X}$ & $\mathrm{X}$ & & & \\
\hline Oligosarcus argenteus Günther, 1864 & & & $\mathrm{X}$ & $\mathrm{X}$ & & \\
\hline Oligosarcus macrolepis (Steindachner, 1877) & & $\mathrm{X}$ & & & & \\
\hline Oligosarcus solitarius Menezes, 1987 & & & $\mathrm{X}$ & & & \\
\hline Piabina argentea Reinhardt, 1867 & $\mathrm{X}$ & & $\mathrm{X}$ & $\mathrm{X}$ & & $\mathrm{X}$ \\
\hline Psellogrammus kennedyi (Eigenmann, 1903) & $\mathrm{X}$ & & & $\mathrm{X}$ & & \\
\hline Rachoviscus graciliceps Weitzman \& Cruz, 1981 & & $\mathrm{X}$ & $\mathrm{X}$ & & & \\
\hline Tetragonopterus chalceus Spix \& Agassiz, 1829 & $\mathrm{X}$ & & & $\mathrm{X}$ & & \\
\hline Lignobrycon myersi (Miranda Ribeiro, 1956) & & $\mathrm{X}$ & & & & \\
\hline Triportheus signatus (Garman, 1890) & & $\mathrm{X}$ & & & & \\
\hline Acestrorhynchus falcatus (Bloch, 1794) & $\mathrm{X}$ & $\mathrm{X}$ & & & & \\
\hline Acestrorhynchus lacustris (Lütken, 1875) & $\mathrm{X}$ & $\mathrm{X}$ & & $\mathrm{X}$ & & \\
\hline Erythrinus erythrinus (Bloch \& Schneider, 1801) & & $\mathrm{X}$ & & & & \\
\hline Hoplerythrinus unitaeniatus (Spix \& Agassiz, 1829) & $\mathrm{X}$ & $\mathrm{X}$ & & $\mathrm{X}$ & & $\mathrm{X}$ \\
\hline Hoplias brasiliensis (Spix \& Agassiz, 1829) & $X$ & $\mathrm{X}$ & & & & \\
\hline Hoplias malabaricus (Bloch, 1794) & $\mathrm{X}$ & $\mathrm{X}$ & $\mathrm{X}$ & $\mathrm{X}$ & $\mathrm{X}$ & $\mathrm{X}$ \\
\hline SILURIFORMES & & & & & & \\
\hline Copionodon lianae Campanario \& de Pinna, 2000 & $\mathrm{X}$ & & & & & \\
\hline Copionodon orthiocarinatus de Pinna, 1992 & $\mathrm{X}$ & & & & & \\
\hline Copionodon pecten de Pinna, 1992 & $\mathrm{X}$ & & & & & \\
\hline
\end{tabular}




\begin{tabular}{|c|c|c|c|c|c|c|}
\hline \multirow{3}{*}{ SPECIES } & \multicolumn{6}{|c|}{ ECOREGIONS } \\
\hline & \multicolumn{3}{|c|}{ NMAF } & \multirow{2}{*}{ São Francisco } & \multirow{2}{*}{ Paraíba do Sul } & \multirow{2}{*}{ Upper Paraná } \\
\hline & NG & CG & SG & & & \\
\hline Copionodon sp. & $\mathrm{X}$ & & & & & \\
\hline Glaphyropoma rodriguesi de Pinna, 1992 & $\mathrm{X}$ & & & & & \\
\hline Glaphyropoma spinosum Bichuette, de Pinna \& Trajano, 2008 & $\mathrm{X}$ & & & & & \\
\hline Ituglanis agreste Lima, Neves \& Campos-Paiva, 2013 & & $\mathrm{X}$ & & & & \\
\hline Ituglanis paraguassuensis Campos-Paiva \& Costa, 2007 & $\mathrm{X}$ & & & & & \\
\hline Trichomycterus bahianus Costa, 1992 & & $\mathrm{X}$ & & & & \\
\hline Trichomycterus itacambirussu Triques \& Vono, 2004 & & $\mathrm{X}$ & & & & \\
\hline Trichomycterus jequitinhonhae Triques \& Vono, 2004 & & $\mathrm{X}$ & & & & \\
\hline Trichomycterus landinga Triques \& Vono, 2004 & & $\mathrm{X}$ & & & & \\
\hline Trichomycterus payaya Sarmento-Soares, Zanata \& Martins-Pinheiro, 2011 & $\mathrm{X}$ & & & & & \\
\hline Trichomycterus pradensis Sarmento-Soares, Martins-Pinheiro, Aranda \& Chamon, 2005 & & & $\mathrm{X}$ & & & \\
\hline Trichomycterus tete Barbosa \& Costa, 2011 & & $\mathrm{X}$ & & & & \\
\hline Trichogenes claviger de Pinna, Helmer, Britski \& Nunes, 2010 & & & $\mathrm{X}$ & & & \\
\hline Aspidoras maculosus Nijssen \& Isbrücker, 1976 & $\mathrm{X}$ & & & & & \\
\hline Aspidoras psammatides Britto, Lima \& Santos, 2005 & $\mathrm{X}$ & & & & & \\
\hline Aspidoras sp. 1 & $\mathrm{X}$ & & & & & \\
\hline Aspidoras sp. 2 & $\mathrm{X}$ & & & & & \\
\hline Aspidoras sp. 3 & $\mathrm{X}$ & & & & & \\
\hline Aspidoras sp. 4 & $\mathrm{X}$ & & & & & \\
\hline Callichthys callichthys (Linnaeus, 1758) & $\mathrm{X}$ & $\mathrm{X}$ & & & & \\
\hline Corydoras nattereri Steindachner, 1876 & & & $\mathrm{X}$ & & $\mathrm{X}$ & \\
\hline Corydoras sp. & & & & & & \\
\hline Hoplosternum littorale (Hancock, 1828) & $\mathrm{X}$ & $\mathrm{X}$ & & $\mathrm{X}$ & & \\
\hline Scleromystax prionotos (Nijssen \& Isbrücker, 1980) & & & $\mathrm{X}$ & & & \\
\hline Pareiorhaphis bahianus (Gosline, 1947) & & $\mathrm{X}$ & & & & \\
\hline Pareiorhaphis lophia Pereira \& Zanata, 2014 & $\mathrm{X}$ & & & & & \\
\hline Pareiorhaphis proskynita Pereira \& Britto, 2012 & & & $\mathrm{X}$ & & & \\
\hline Pareiorhaphis scutula Pereira, Vieira \& Reis, 201 & & & $\mathrm{X}$ & & & \\
\hline Pareiorhaphis sp. 1 & $\mathrm{X}$ & & & & & \\
\hline Pareiorhaphis sp. 2 & & $\mathrm{X}$ & & & & \\
\hline Pareiorhaphis sp. 3 & & $\mathrm{X}$ & & & & \\
\hline An undescribed loricariid genus & & & $\mathrm{X}$ & & & \\
\hline Hisonotus sp. & & & $\mathrm{X}$ & & & \\
\hline Otothyris travassosi Garavello, Britski \& Schaefer, 1998 & & $\mathrm{X}$ & $\mathrm{X}$ & & & \\
\hline Parotocinclus arandai Sarmento-Soares, Lehmann \& Martins-Pinheiro, 2009 & & & $\mathrm{X}$ & & & \\
\hline Parotocinclus bahiensis (Miranda Ribeiro, 1918) & $\mathrm{X}$ & & & & & \\
\hline Parotocinclus cristatus Garavello, 1977 & & $\mathrm{X}$ & & & & \\
\hline Parotocinclus doceanus (Miranda Ribeiro, 1918) & & & $\mathrm{X}$ & & & \\
\hline Parotocinclus jimi Garavello, 1977 & & $\mathrm{X}$ & & & & \\
\hline Parotocinclus planicauda Garavello \& Britski, 2003 & & & $\mathrm{X}$ & & & \\
\hline Parotocinclus sp. 1 & $\mathrm{X}$ & & & & & \\
\hline Parotocinclus sp. 2 & & $\mathrm{X}$ & & & & \\
\hline Parotocinclus sp. 3 & $\mathrm{X}$ & & & & & \\
\hline Parotocinclus sp. 4 & & $\mathrm{X}$ & & & & \\
\hline Loricariichthys castaneus (Castelnau, 1855) & & & $\mathrm{X}$ & & $\mathrm{X}$ & \\
\hline Hypostomus chrysostiktos Birindelli, Zanata \& Lima, 2007 & $\mathrm{X}$ & & & & & \\
\hline Hypostomus jaguar Zanata, Sardeiro \& Zawadzki, 2013 & $\mathrm{X}$ & & & & & \\
\hline Pogonopoma wertheimeri (Steindachner, 1867) & & & $\mathrm{X}$ & & & \\
\hline Delturus angulicauda (Steindachner, 1877) & & & $\mathrm{X}$ & & & \\
\hline Delturus brevis Reis \& Pereira, 2006 & & $\mathrm{X}$ & & & & \\
\hline
\end{tabular}




\begin{tabular}{|c|c|c|c|c|c|c|}
\hline \multirow{3}{*}{ SPECIES } & \multicolumn{6}{|c|}{ ECOREGIONS } \\
\hline & \multicolumn{3}{|c|}{ NMAF } & \multirow{2}{*}{ São Francisco } & \multirow{2}{*}{ Paraíba do Sul } & \multirow{2}{*}{ Upper Paraná } \\
\hline & NG & $\mathrm{CG}$ & SG & & & \\
\hline Delturus carinotus (La Monte, 1933) & & & $\mathrm{X}$ & & & \\
\hline Microglanis pataxo Sarmento-Soares, Martins-Pinheiro, Aranda \& Chamon, 2006 & & & $\mathrm{X}$ & & & \\
\hline Acentronichthys leptos Eigenmann \& Eigenmann, 1889 & & $\mathrm{X}$ & $\mathrm{X}$ & & & \\
\hline Cetopsorhamdia iheringi Schubart \& Gomes, 1959 & $\mathrm{X}$ & $\mathrm{X}$ & & $\mathrm{X}$ & & $\mathrm{X}$ \\
\hline Imparfinis minutus (Lütken, 1874) & & $\mathrm{X}$ & $\mathrm{X}$ & $\mathrm{X}$ & & \\
\hline Pimelodella harttii (Steindachner, 1877) & & $\mathrm{X}$ & $\mathrm{X}$ & & $\mathrm{X}$ & \\
\hline Pimelodella itapicuruensis Eigenmann, 1917 & $\mathrm{X}$ & & & & & \\
\hline Pimelodella lateristriga (Lichtenstein, 1823) & & $\mathrm{X}$ & $\mathrm{X}$ & & $\mathrm{X}$ & \\
\hline Pimelodella sp. 1 & $\mathrm{X}$ & & & & & \\
\hline Pimelodella sp. 2 & & $\mathrm{X}$ & & & & \\
\hline Rhamdia quelen (Quoy \& Gaimard, 1824) & $\mathrm{X}$ & $\mathrm{X}$ & $\mathrm{X}$ & $\mathrm{X}$ & $\mathrm{X}$ & $\mathrm{X}$ \\
\hline Rhamdia sp. & & $\mathrm{X}$ & & & & \\
\hline Rhamdiopsis krugi Bockmann \& Castro, 2010 & $\mathrm{X}$ & & & & & \\
\hline Pimelodus sp. & $\mathrm{X}$ & & & & & \\
\hline Steindachneridion doceanum (Eigenmann \& Eigenmann, 1889) & & & $\mathrm{X}$ & & & \\
\hline Kalyptodoras bahiensis Higuchi, Britski \& Garavello, 1990 & $\mathrm{X}$ & & & & & \\
\hline Wertheimeria maculata Steindachner, 1877 & & $\mathrm{X}$ & & & & \\
\hline Glanidium botocudo Sarmento-Soares \& Martins-Pinheiro, 2013 & & & $\mathrm{X}$ & & & \\
\hline Trachelyopterus galeatus (Linnaeus, 1766) & $\mathrm{X}$ & $\mathrm{X}$ & & & & \\
\hline Trachelyopterus striatulus (Steindachner, 1877) & & & $\mathrm{X}$ & & $\mathrm{X}$ & $\mathrm{X}$ \\
\hline Pseudauchenipterus affinis (Steindachner, 1877) & & & $\mathrm{X}$ & & & \\
\hline Pseudauchenipterus jequitinhonhae (Steindachner, 1877) & & $\mathrm{X}$ & & & & \\
\hline GYMNOTIFORMES & & & & & & \\
\hline Gymnotus bahianus Campos-da-Paz \& Costa, 1996 & & $\mathrm{X}$ & & & & \\
\hline Gymnotus carapo Linnaeus, 1758 & $\mathrm{X}$ & $\mathrm{X}$ & $\mathrm{X}$ & $\mathrm{X}$ & $\mathrm{X}$ & $\mathrm{X}$ \\
\hline Gymnotus pantherinus (Steindachner, 1908) & & & $\mathrm{X}$ & & & \\
\hline Gymnotus sp. & & $\mathrm{X}$ & & & & \\
\hline CYPRINODONTIFORMES & & & & & & \\
\hline Anablepsoides bahianus (Huber, 1990) & $\mathrm{X}$ & & & & & \\
\hline Cynolebias itapicuruensis Costa, 2001 & $\mathrm{X}$ & & & & & \\
\hline Cynolebias vazabarrisensis Costa, 2001 & $\mathrm{X}$ & & & & & \\
\hline Simpsonichthys bokermanni (Carvalho \& Da Cruz, 1987) & & $\mathrm{X}$ & & & & \\
\hline Simpsonichthys perpendicularis Costa, Nielsen \& de Luca, 2001 & & $\mathrm{X}$ & & & & \\
\hline Simpsonichthys rosaceus Costa, Nielsen \& de Luca, 2001 & & $\mathrm{X}$ & & & & \\
\hline Simpsonichthys suzarti Costa, 2004 & & $\mathrm{X}$ & & & & \\
\hline Pamphorichthys hollandi (Henn, 1916) & $\mathrm{X}$ & & & $\mathrm{X}$ & & \\
\hline Phalloceros elachistos Lucinda, 2008 & & & $\mathrm{X}$ & & & \\
\hline Phalloceros ocellatus Lucinda, 2008 & & $\mathrm{X}$ & $\mathrm{X}$ & & & \\
\hline Phalloceros sp. & & $\mathrm{X}$ & $\mathrm{X}$ & & & \\
\hline Phalloptychus eigenmanni Henn, 1916 & $\mathrm{X}$ & & & & & \\
\hline Poecilia vivipara Bloch \& Schneider, 1801 & $\mathrm{X}$ & $\mathrm{X}$ & $\mathrm{X}$ & $\mathrm{X}$ & $\mathrm{X}$ & $\mathrm{X}$ \\
\hline SYNBRANCHIFORMES & & & & & & \\
\hline Synbranchus marmoratus Bloch, 1795 & & & & & & \\
\hline PERCIFORMES & & & & & & \\
\hline Australoheros facetus (Jenyns, 1842) & & & $\mathrm{X}$ & & & \\
\hline Cichlasoma sanctifranciscense Kullander, 1983 & $\mathrm{X}$ & & & $\mathrm{X}$ & & \\
\hline Crenicichla lacustris (Castelnau, 1855) & & & $\mathrm{X}$ & & $\mathrm{X}$ & \\
\hline Crenicichla lepidota Heckel, 1840 & $\mathrm{X}$ & & & $\mathrm{X}$ & & \\
\hline Crenicichla mucuryna Ihering, 1914 & & & $\mathrm{X}$ & & & \\
\hline
\end{tabular}




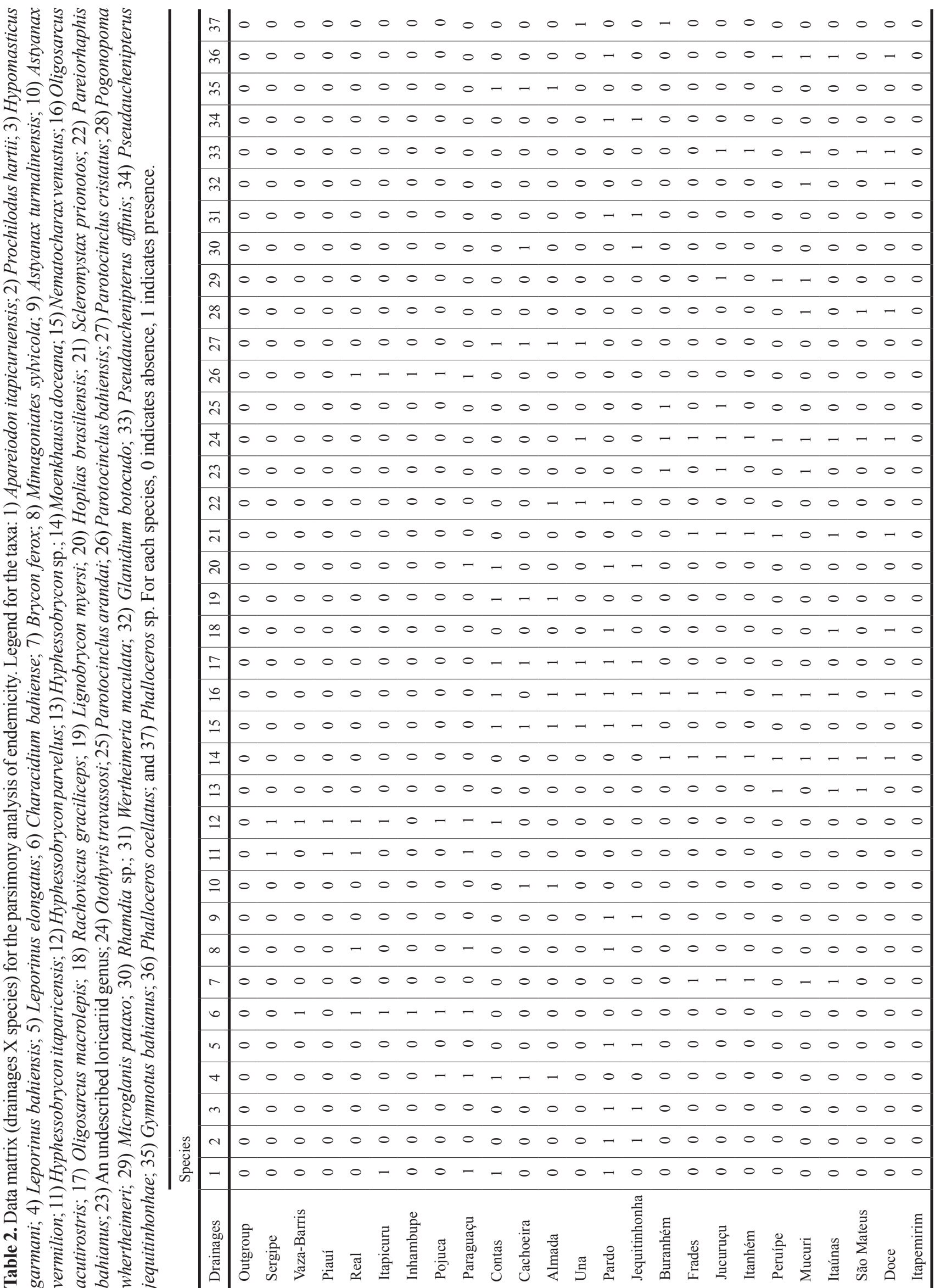


Relationships among drainages of the Northeastern Mata Atlântica freshwater ecoregion and its shared fish fauna. PAE identified five equally parsimonious trees with length of 71 steps. The strict consensus diagram [Consistency Index $(\mathrm{CI})=0.521$; Retention Index $(\mathrm{RI})=0.709]$ shows two main groups of basins within the NMAF ecoregion, which were defined based on their shared species of freshwater fishes (Fig. 2). These groups were herein denominated: North Group and Central-South Group. The Central-South Group shows a basal polytomy composed by two groups (which were denominated as Central Group and South Group) plus the rio Itapemirim basin (Figs. 1-2). The consensus represents a combination of five competing hypotheses regarding the relationships among the some basins within the North Group, South Group, and also the position of rio Itapemirim basin.

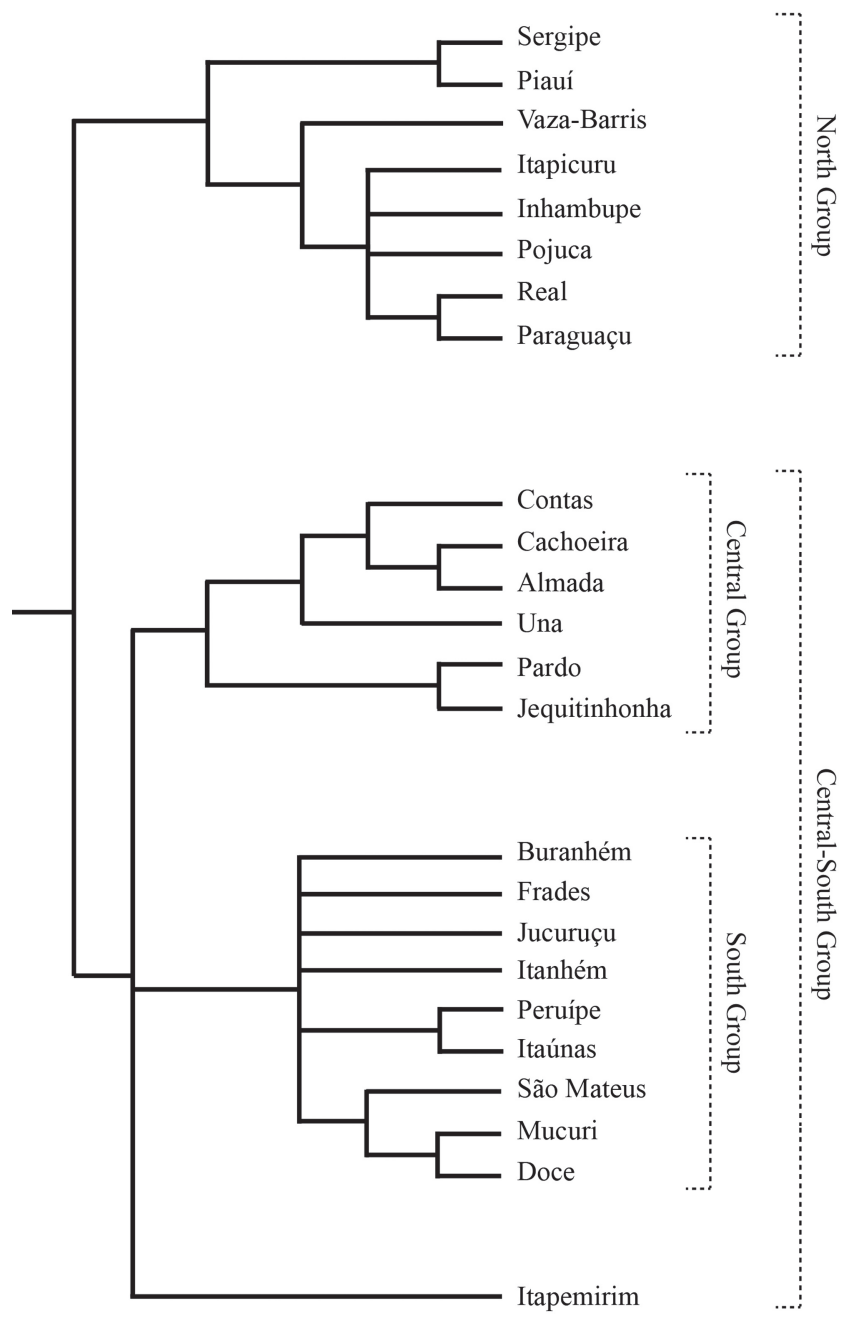

Fig. 2. Area diagram depicting relationships among the 24 coastal drainages analyzed, obtained by parsimony analysis of endemicity based on freshwater fishes. The topology represents the strict consensus of five equally parsimonious trees obtained through a heuristic search (length $=71$ steps, $\mathrm{CI}=0.521, \mathrm{RI}=0.709$ ).
North Group. Included are eight drainages (from north to south: Sergipe, Vaza-Barris, Piauí, Real, Itapicuru, Inhambupe, Pojuca, and Paraguaçu; Figs. 1-2, Table 3) situated in the northernmost portion of the NMAF ecoregion. The North Group is supported by the shared presence of only one species, Hyphessobrycon parvellus, originally described from the rio Pojuca basin and recorded herein to all drainages of the group with the exception of rio Inhambupe. This absence may be due to undersampling. Outside of the North Group drainages, H. parvellus also occurs in the rio de Contas basin (northernmost member of the Central Group). Although the rio de Contas drainage shares a high number of species with remaining rivers of the Central Group, especially Almada and Cachoeira, it also shares a few other species with basins of the North Group. Within the North Group, a close relationship between the rio Sergipe and rio Piauí basins is supported by the presence of Hyphessobrycon itaparicensis and a close relationship between the VazaBarris, Real, Itapicuru, Inhambupe, Pojuca, and Paraguaçu basins is supported by the shared presence of Characidium bahiense. According to the consensus diagram, the Real, Itapicuru, Inhambupe, Pojuca, and Paraguaçu drainages are more closely related to each other, based on the presence of Parotocinclus bahiensis. Hyphessobrycon itaparicensis and Mimagoniates sylvicola supported a close relationship among Real and Paraguaçu basins.

Central-South Group. Included are 16 drainages (from north to south: rio de Contas, Cachoeira, Almada, Una, Pardo, Jequitinhonha, Buranhém, Frades, Jucuruçu, Itanhém, Peruípe, Mucuri, Itaúnas, São Mateus, Doce, and Itapemirim; Figs. 1-2, Table 3). The Central-South Group is supported by the shared presence of only one species, Oligosarcus acutirostris, described originally from the rio Itapemirim basin, and not recorded herein from only three drainages of the group (Cachoeira, Itanhém, and São Mateus). The species absence in these three rivers may be due to undersampling, once the known distribution of the species includes rivers from eastern coastal rivers between states of Bahia and Espírito Santo (Menezes, 2003). Within the Central-South Group, there are two groups, Central Group and South Group, which are described below, in addition to the Itapemirim basin. According to the consensus, no species supports a closer relationship of the rio Itapemirim with one or the other group within the Central-South Group. However, the rio Itapemirim shares Scleromystax prionotos (originally described from the rio Doce basin) with the South Group, fact that could indicate a similarity of fauna between the rio Itapemirim and the group formed by Buranhém, Frades, Jucuruçu, Itanhém, Peruípe, Mucuri, Itaúnas, São Mateus, and Doce rivers.

Central Group. Included are six drainages (from north to south: rio de Contas, Cachoeira, Almada, Una, Pardo, and Jequitinhonha; Figs. 1-2, Table 3) situated in the central portion of the NMAF ecoregion. Two species shared exclusively by these drainages support this group: 
Nematocharax venustus and Oligosarcus macrolepis. Both species were described from the rio Jequitinhonha basin and were collected in all Central Group drainages. Within the Central Group, also there are two groups: one including rio de Contas, Cachoeira, Almada, and Una basins, and the other formed by the Pardo and Jequitinhonha basins. The former group is supported by the presence of Parotocinclus cristatus, described from the rio Almada basin. Within this group, a close relationship between the rio de Contas, Almada, and Cachoeira basins is supported by the shared occurrences of Leporinus bahiensis, Lignobrycon myersi, and Gymnotus bahianus. According to the results, the Almada and Cachoeira drainages are more closely related to each other, based on the shared presence of Astyanax vermilion. Of all the proposed relationships among rivers, that of Pardo and Jequitinhonha had the strongest support according to the PAE results, with seven shared species, six of them endemic to those basins (Astyanax turmalinensis, Hypomasticus garmani, Leporinus elongatus, Prochilodus hartii, Pseudachenipterus jequitinhonhae, and Wertheimeria maculata) and only one non-endemic, Hoplias brasiliensis, described from the rio Paraguaçu basin (North Group).

Table 3. Biogeographic groups of drainages of Northeastern Mata Atlântica freshwater ecoregion and the species used to support them, as determined by parsimony analysis of endemism.

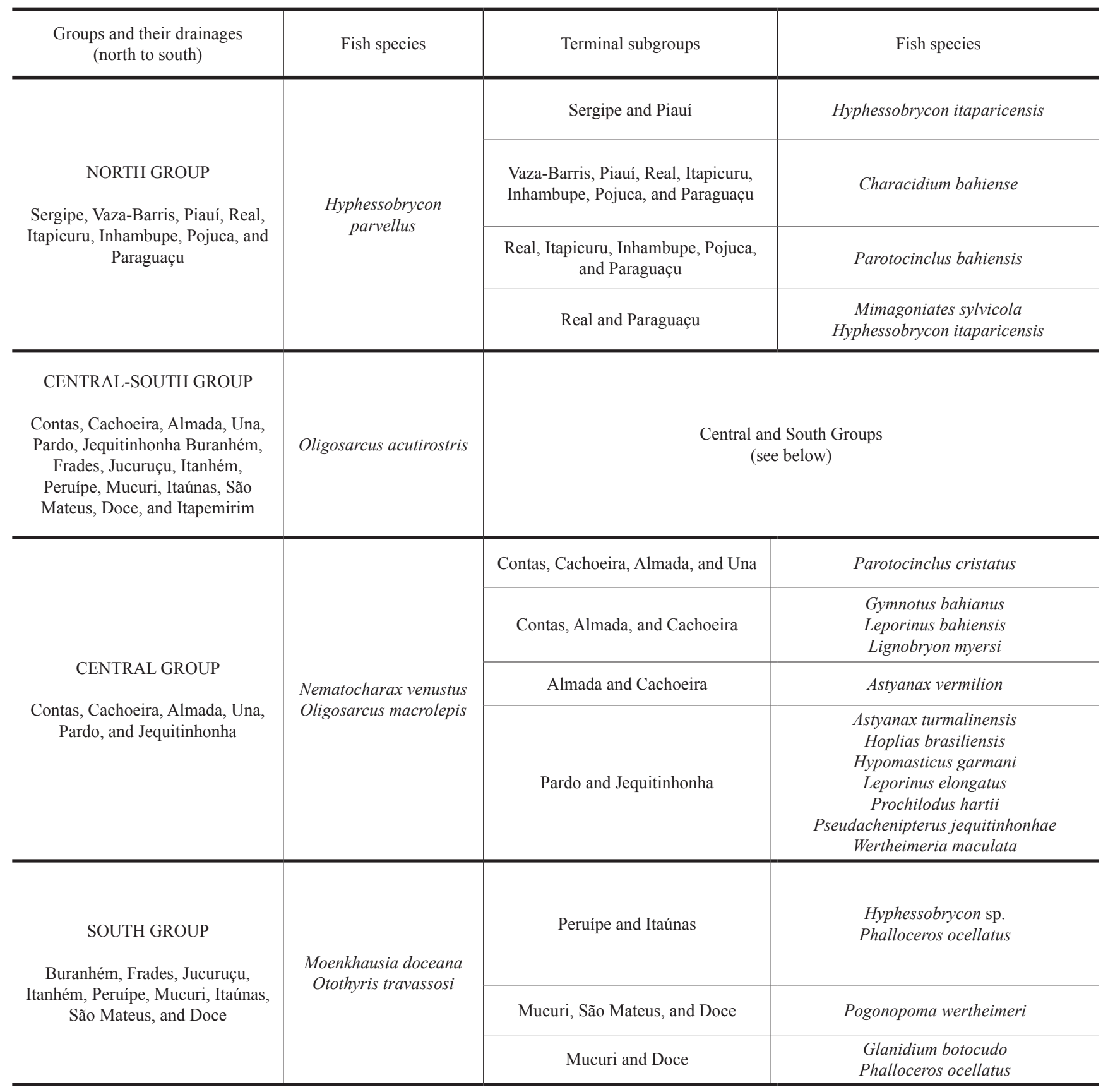


South Group. Included are nine drainages (from north to south: Buranhém, Frades, Jucuruçu, Itanhém, Peruípe, Mucuri, Itaúnas, São Mateus, and Doce; Figs. 1-2, Table 3 ), situated in the southernmost portion of the NMAF ecoregion, and supported by two species: Moenkhausia doceana, described from the rio Doce basin, and Otothyris travassosi, described from the rio São Mateus basin. Both species were collected in all drainages of the South Group and $O$. travassosi was also collected in the rio Una basin (Central Group). According to the consensus diagram, the relationships among the drainages included in the South Group are not clear, although there are species shared exclusively by some rivers of the South Group (e.g., Brycon ferox, shared only by Frades, Jucuruçu, Itanhaém, Mucuri, and Itaúnas basins, Microglanis pataxo, shared only by Jucuruçu, Peruípe, and Mucuri, Parotocinclus arandai, shared only by Buranhém and Jucuruçu, and an undescribed loricariid genus, shared only by Buranhém, Jucuruçu, and Mucuri). According to the consensus diagram, the Mucuri, São Mateus, and Doce drainages are more closely related, based on the sharing of Pogonopoma wertheimeri. Within this group, a close relationship between the Mucuri and Doce drainages is supported by the shared presence of two species: Glanidium botocudo and Phalloceros ocellatus. Hyphessobrycon sp. and Phalloceros ocellatus supported the close relationship among Peruípe and Itaúnas basins.

Of the 37 species included in the biogeographic analysis, only three were restricted to the basins of the North Group, 14 were restricted to the Central Group, and ten were restricted to the South Group (Table 1). Five species were shared by the rivers of the North and Central Groups, five were shared by the Central and South Groups, and no species was shared by North and South Groups. Therefore, the rivers of the Central Group appear to be intermediate between basins situated to the north and to the south in the NMAF ecoregion, not only from a geographic point of view but also in terms of their ichthyofaunal composition.

A gradual change in fish composition with latitude was observed, both at the species level and for more inclusive taxonomic categories. The number of species of the order Siluriformes within the groups increases from north to south. Thus, of the species included in the biogeographic analysis, members of Siluriformes represent 7\% in the North Group, $36 \%$ in the Central Group, and 57\% in the South Group. An opposite trend was observed for members of the order Characiformes, which is more representative in Central Group and North Group (with $80 \%$ and $30 \%$, respectively) than South Group (25\%). These results are in agreement with published information about various rivers included in South Group, where a higher proportion of Siluriformes was observed (e.g., Sarmento-Soares \& Martins-Pinheiro, 2009, 2012; Sarmento-Soares et al. 2007, 2008, 2009b).

Brazilian eastern coastal biogeographical units were previously proposed, on the base of fish species, for some drainages included in the area (Géry, 1969; Vari, 1988) or most of them (Menezes, 1988; Weitzman et al., 1988;
Bizerril, 1994). According to Jacobina et al. (2009), the presence of endemic species in some of the drainages of eastern Brazil indicates the existence of biogeographic units or subprovinces in the area, though the pattern of subdivision is complex. Two subprovinces in the Brazilian eastern coastal area were proposed by Bizerril (1994), and termed "subprovince of the southeast coast" (from 'Mountainous region' of Rio de Janeiro State to the south of Santa Catarina State) and "subprovince of the east coast" (from coastal basins in Bahia south of the rio São Francisco outlet to rio Paraíba do Sul basin = very similar to NMAF ecoregion). In agreement with Bizerril (1994), our results indicate that the eastern basins represent a biogeographic unit when compared to the remaining hydrographic basins of Brazil, but with peculiarities in its ichthyofaunistic composition that warrant the recognition of subregions. Thus, the NMAF ecoregion appears to be heterogeneous, with two main groups of basins (North and Central-South) and groups (Central and South), as described above, plus the rio Itapemirim basin.

Fish fauna shared with adjacent ecoregions and comments about geomorphological factors influencing local ichthyofaunistic composition. Of the 192 species listed in this study for the NMAF ecoregion, 37 are known also to occur in adjacent ecoregions (i.e., São Francisco, Paraíba do Sul, and Upper Paraná, Fig. 1), not considering species that are widely distributed and/or taxonomically complex. Of those 37 species, one is shared with these three adjacent ecoregions, 21 are shared only São Francisco ecoregion, seven only with Paraíba do Sul, and nine with Upper Paraná ecoregion (six also shared with São Francisco, two with Paraíba do Sul, and one with both; Table 1). Of the 21 species shared only between the drainages of the NMAF ecoregion and rio São Francisco basin, 12 occur only in the North Group, five occur in both the North and Central Groups, one only in the Central Group, one in both the Central and South Groups, and two only in the South Group. Bizerril (1994) noted a gradual change in the ichthyofaunal composition of the coastal Brazilian drainages, especially when going from south to north, which was characterized by an increase in endemism in parallel with increasing taxonomic affinity with the rio São Francisco basin. Thus, our results agree with the latitudinal rise of ichthyofaunal similarity with the São Francisco basin, as suggested by Bizerril (1994) when discussing his "subprovince of the east coast".

Of the studied drainages, the rio Itapicuru basin (North Group) has the greatest ichthyofaunal similarity to the rio São Francisco basin. Of the 21 species shared only between the drainages of the NMAF ecoregion and rio São Francisco basin, 13 occur in the rio Itapicuru basin and four of them are exclusive of these two basins (Curimatella lepidura, Hyphessobrycon micropterus, Leporinus reinhardti, and Prochilodus costatus). Leporinus taeniatus was described from the rio São Francisco and was considered as endemic 
to this basin (Britski \& Garavello, 2003). However, in this study, we recorded specimens of L. taeniatus also to the rio Itapicuru and rio Vaza-Barris basins. These records reinforce, in part, the hypothesis of Costa (2001), who suggested the Itapicuru and Vaza-Barris basins as possibly old tributaries of an ancient basin that included the main course of the middle rio São Francisco. The Atlantic outlet of the rio Vaza-Barris is just north of that of the rio Real, and its headwaters about those of the Itapicuru and right bank tributaries of the lower rio São Francisco. Costa's (2001) hypothesis was primarily based on the occurrence of a Cynolebias Steindachner clade in the upper sections of the Itapicuru and Vaza-Barris basins (C. itapicuruensis and $C$. vazabarrisensis, respectively) that is closely related to a clade endemic to the middle rio São Francisco basin. Furthermore, the similarity between the Itapicuru and São Francisco drainages shown in this study reinforces the idea that river capture events occurred between these two river basins, as proposed by Haseman (1912) and, more recently, by Costa (2001).

Of the five species shared between the rio São Francisco basin and North plus Central Groups, three, Cichlasoma sanctifranciscense, Pamphorichthys hollandi, and Phenacogaster franciscoensis, occur in North Group and in the rio de Contas basin, the northernmost member of the Central Group which also shares a few species with basins of the North Group. Thus, the southernmost limit of distribution of these species is the rio de Contas, which seems to be the drainage from which the taxonomic affinity with the rio São Francisco basin clearly decreases. Acinocheirodon melanogramma is the unique species shared only between a member of the Central Group (rio Jequitinhonha) and rio São Francisco basin. There is some geomorphological evidence indicating ancient drainage connection among rio Jequitinhonha and rio São Francisco basins, mainly based on the large number of wind gaps (i.e., now dry former water courses) border the Espinhaço complex (Saadi, 1998). According to this author, the great curvature of the rio Itacambiruçu (tributary of the Jequitinhonha that begins flowing in a northwesterly direction before curving northeastward and eventually southeastward towards its confluence with the Jequitinhonha), for example, may correspond to a mesozoic capture of an ancient tributary of the rio São Francisco basin. However, these geomorphological events are very ancient and, in fact, a high number of shared species is not expected.

Only four species were shared by drainages of the South Group and the rio São Francisco (Imparfinis minutus, Lepidocharax burnsi, Oligosarcus argenteus, and Prochilodus vimboides), which is a result possibly related to the isolation of those drainages from headwaters of the latter. The isolation of headwaters of the São Francisco is a consequence of very ancient orogenies (Potter, 1997) that contributed to the formation of the Serra da Canastra at one end of the Espinhaço complex (Alkmim et al., 2007).
Compared to the North and Central Groups, the drainages of the South Group have a higher faunal similarity with the rio Paraíba do Sul ecoregion. Of the seven species shared only between the drainages of the NMAF ecoregion and rio Paraíba do sul basin, five are distributed only in the South Group, two occur in both the South and Central Groups, and none in the North Group. Furthermore, the close relationship among rivers of the South Group and Paraíba do Sul basin is also indicated in the phylogenetic study proposed by Quevedo \& Reis (2002). According to their hypothesis for the armored catfish genus Pogonopoma Regan, the species $P$. wertheimeri, endemic to Doce, Mucuri, and São Mateus basins, is the sister group of $P$. parahybae (Steindachner), endemic to rio Paraíba do Sul basin.

Menezes (1972) and Bizerril (1994) noted higher similarity among fish faunas of the coastal basins in eastern Brazil and rio Paraná basin than between the former and rio São Francisco, a conclusion distinct from that obtained herein for the majority of the rivers included in the NMAF ecoregion. Those previous analyses focused on the coastal drainages situated more to the south and did not include the rivers located further north in the ecoregion (e.g., Itapicuru). According to our results, these northern rivers have a higher number of species in common with the São Francisco basin, and are distinct from the southern rivers in species composition. In fact, of the 192 species listed in this study for the NMAF ecoregion, only nine are known to occur in the rio Paraná basin, and all of them also occur in the rio São Francisco and/or rio Paraíba do Sul basins. The frequencies of shared species observed between the drainages of the NMAF ecoregion and the São Francisco and Paraná basins, excluding species that are taxonomically complex and widely distributed, are about $14 \%$ and $5 \%$, respectively, indicating a much higher faunal similarity between the coastal drainages of the ecoregion analyzed and the São Francisco basin. Thus, the ichthyofaunistic similarity of the Paraná and basins of eastern Brazil becomes stronger further south. Faunistic interchanges may have occurred between the rio Paraíba do Sul basin, one of the largest eastern rivers, and the rio Tietê, a tributary of the rio Paraná (Menezes, 1972; Ribeiro, 2006; Serra et al., 2007). Hypotheses explaining that interchange are discussed by various authors (e.g., Ab'Saber, 1957; Armbruster, 1998; Quevedo \& Reis, 2002; Ribeiro, 2006), and are beyond the scope of this study.

\section{Biogeographic aspects in light of phylogenetic information for fishes occurring in drainages the NMAF ecoregion and patterns of biogeographic relationships.} One of the few phylogenetic studies available that allows for comparisons to the framework proposed here is that of Costa (2003). According to his hypothesis for the annual fish genus Simpsonichthys Carvalho, the species S. rosaceus (endemic to the rio Pardo basin) and S. perpendicularis (endemic to the rio Jequitinhonha basin) form a clade sister 
to $S$. bokermanni (known only from rio Cachoeira basin). This phylogenetic hypothesis indicates a close relationship between the rios Pardo and Jequitinhonha basins and between these drainages and rio Cachoeira (all members of the Central Group). The close relationship of those rivers is congruent with the pattern suggested by the PAE results (Fig. 2). No other phylogenetic hypothesis is available at this time to verify the remaining relationships proposed herein among rivers of NMAF ecoregion. However, according to Ribeiro (2006), phylogenetic patterns indicate a close biotic relationship between the rivers that flow into the Atlantic Ocean and those on the adjacent upland crystalline shield (specifically the rios Paraná and São Francisco basins). This suggests that cladogenetic events between the two groups of rivers have occurred continuously over long periods of time, and have given rise to similar sister group phylogenetic patterns.

The presence of basal taxa in some of the drainages of NMAF ecoregion indicates that part of the fauna of this region is formed by taxa reminiscent of an ancient biogeographic history, as previously stated by Ribeiro (2006) for other eastern Brazilian drainages (i.e., his "Pattern A" drainages). Those taxa are depauperate in species and have restricted geographical distributions when compared to their sister groups, a phenomenon previously discussed by Stiassny \& de Pinna (1994) for fishes in general, and, according to Ribeiro (2006), strikingly prevalent among freshwater fishes and some coastal drainages of eastern Brazil. The examples given by Ribeiro (2006) for sister group relationships between subfamilies are mostly based on groups occurring in or endemic to the northeastern coastal drainages of Brazil, reinforcing the interesting nature of the biogeographical history of those drainages. One of his examples refers to the trichomycterid subfamily Copionodontinae (with two genera and five species endemic to the upper rio Paraguaçu, member of North Group), which forms a monophyletic group with Trichogeninae (with one genus and two species, Trichogenes longipinnis Britski \& Ortega, endemic to coastal drainages in northern São Paulo State, and T. claviger, endemic to rio Itapemirim basin, member of Central-South Group). This monophyletic group, in turn, is considered sister group of the remaining trichomycterids (more than 200 described species widely distributed from Patagonia to Panama; Bichuette et al., 2008). Another example involves the loricariid subfamily Delturinae, represented here by solely one genus, Delturus Eigenmann \& Eigenmann, and three species, D. angulicauda (endemic to the rio Mucuri basin, South Group), D. brevis (known only from the rio Jequitinhonha basin, Central Group), and D. carinotus (endemic to the rio Doce drainage, South Group). The subfamily Delturinae, which includes Delturus plus Hemipsilichthys Eigenmann \& Eigenmann, is considered sister group of all remaining loricariids except Lithogenes Eigenmann (Reis et al., 2006), with more than 870 described species widely distributed in South and Central America (Eschmeyer \& Fong, 2014). Also, another example of the Pattern A involves the family Doradidae, represented here by Kalyptodoras bahiensis (endemic to the rio Paraguaçu basin, North Group) and Wertheimeria maculata (known only from the Jequitinhonha and Pardo drainages, Central Group). Recently, Mariangeles et al. (2013), proposed the "Wertheimeria clade" to include the two cited doradid species plus Franciscodoras marmoratus (Lütken), a species endemic to the rio São Francisco basin. In their analysis, the "Wertheimeria clade" is the sister group of a much larger (ca. 65 species) and more widely distributed group of doradids, their "Clade 2".

Taxa with an intermediate biogeographic history resulting from continued faunal interchanges between the upland Brazilian crystalline shield and coastal rivers throughout the Tertiary (Paleogene and Neogene) represent "Pattern B" of Ribeiro (2006). Those taxa are exemplified in the studied drainages by Kolpotocheirodon Malabarba \& Weitzman, Lepidocharax Ferreira, Menezes \& QuagioGrassiotto, Lignobrycon Eigenmann, Mimagoniates Regan, Pogonopoma, and Scleromystax Günther. The cheirodontinae Kolpotocheirodon is the most basal genus of the tribe Compsurini and includes only two species, $K$. figueiredoi (known only from the type locality, a tributary of the upper portion of rio Paraguaçu, North Group) and $K$. theloura Malabarba \& Weitzman (endemic to the uppermost tributaries of the São Francisco and Paraná rivers; Malabarba et al., 2004). The basal and recently described Stevardiinae genus Lepidocharax includes only two species: L. burnsi (endemic to the tributaries of the upper rio São Francisco and Rio Doce basins) and L. diamantina (endemic to the rio Paraguaçu basin; Ferreira et al., 2011). For further information on the remaining examples of "Pattern B" taxa, see Ribeiro (2006: 236-238).

Another putative example involving sister group relationships between coastal and continental rivers is that of Hasemania piatan, a recently described species from a tributary of the upper rio de Contas basin (Zanata \& Serra, 2010). Of its seven congeners, four have a limited distribution in the rio Paraná and rio São Francisco basins, one is from an unknown locality in the State of Goiás, one is from a tributary of the upper rio Juruena (tributary of the upper rio Tapajós basin), and one from upper rio Tocantins basin. The monophyly of Hasemania Ellis, traditionally based mainly on the lack of an adipose fin, has been questioned by several authors. Thus, the efficacy of this group as an example of "Pattern B" of Ribeiro depends on the definition of Hasemania as a monophyletic group.

Ribeiro (2006) applied the term "Pattern C" to distributions that reflect the most recent exchanges between the upland crystalline shield rivers and the adjacent coastal drainages, recognized on the basis of shared species, and, in some cases, of hybrid zones. As stated previously, we found 28 species occurring in drainages of the NMAF ecoregion that are also present in the rio São Francisco (without considering species that are widely distributed and/or taxonomically complex). Most 
of them have a somewhat broad distribution, occurring also in some of the coastal drainages north of the rio São Francisco, and six are shared with the rio Paraná basin. Thus, of the 28 species, Hyphessobrycon micropterus, Lepidocharax burnsi, Oligosarcus argenteus, Prochilodus costatus, Steindachnerina elegans, and Phenacogaster franciscoensis are currently known to occur only in the rio São Francisco basin and some of the coastal drainages studied herein. Those species appear to exemplify "Pattern C" as defined by Ribeiro. In turn, it appears that only Acestrorhynchus lacustris, Cetopsorhamdia iheringi, Hemigrammus marginatus, Hyphessobrycon bifasciatus, Piabina argentea, Prochilodus vimboides, Serrapinnus heterodon, S. piaba, and Trachelyopterus striatulus are shared between the drainages of the NMAF ecoregion and the rio Paraná basin. Those species also exemplify the cited "Pattern C" and most of them are relatively widely distributed, occurring also in the rio São Francisco basin with the exception of $H$. bifasciatus and T. striatulus.

Apart from the fishes broadly shared across continental hydrographic basins, this study reveals a number of species that are shared exclusively by coastal rivers of the NMAF ecoregion, a pattern suggestive of past connections between them. Some of those shared species include Apareiodon itapicuruensis, Astyanax vermilion, Brycon ferox, Characidium bahiense, Mimagoniates sylvicola, Gymnotus bahianus, Hyphessobrycon itaparicensis, H. parvellus, Leporinus bahiensis, Microglanis pataxo, Pareiorhaphis bahianus, Parotocinclus arandai, P. bahiensis, P. cristatus, and Pogonopoma wertheimeri. The widespread geographic ranges of several taxa along neighbouring coastal drainages can be explained by the hypothesis of Weitzman et al. (1988), who proposed that the diversification of the fish faunas of the coastal drainages of South America is related to sea level changes that occurred during the Late Pleistocene. Their explanation agrees with that of Ribeiro (2006).

\section{Acknowledgments}

We would like to thank Adolfo Calor (UFBA), Flávio Lima (UNICAMP), José Birindelli (UEL), Mark Sabaj Pérez (ANSP), and Silvio Nihei (IB-USP) for valuable suggestions on the manuscript. Identification of various taxa had the help of Carine Chamon (UFT), Edson Pereira (PUCRS), Heraldo Britski (MZUSP), José Birindelli (UEL), Marcelo Britto (MNRJ), Mônica Toledo-Piza (USP), and Naércio Menezes (MZUSP). Thanks also to a number of persons who helped during field expeditions. Eduardo G. Baena made the Fig. 1. Adolfo Calor also helped with the Fig. 2. Collecting expeditions were funded by $\mathrm{CNPq}$ (309840/2003-1, 476449/2007-3, and 476495/2010-5), the All Catfish Species Inventory (NSF DEB-0315963), and the South American Characiformes Inventory (FAPESP \# 2011/50282-7, http://www.projeto-saci.com). The senior author had a master's fellowship from CAPES.

\section{Literature Cited}

Abell, R., M. L.Thieme, C. Revenga, M. Bryer, M. Kottelat, N. Bogutskaya, B. Coad, N. Mandrak, S. C. Balderas, W. Bussing, M. L. J. Stiassny, P. Skelton, G. R. Allen, P. Unmack, A. Naseka, R. Ng, N. Sindorf, J. Robertson, E. Armijo, J. V. Higgins, T. J. Heibel, E. Wikramanayake, D. Olson, H. L. López, R. E. Reis, J. G. Lundberg, M. H. S. Pérez \& P. Petry. 2008. Freshwater ecoregions of the world: A new map of biogeographic units for freshwater biodiversity conservation. Bioscience, 58: 403-414.

Ab'Saber, A. N. 1957. O problema das conexões antigas e da separação da drenagem do Paraíba e Tietê. Boletim Paulista de Geografia, 26: 38-49.

Armbruster, J. W. 1998. Phylogenetic relationships of the suckermouth armored catfishes of the Rhinelepis group (Loricariidae: Hypostominae). Copeia, 1998: 620-636.

Bichuette, M. E., M. C. C. de Pinna \& E. Trajano. 2008. A new species of Glaphyropoma: the first subterranean Copionodontinae catfish and the first occurrence of opercular odontodes in the subfamily (Siluriformes: Trichomycteridae). Neotropical Ichthyology, 6: 301-306.

Bizerril, C. R. S. F. 1994. Análise taxonômica e biogeográfica da ictiofauna de água doce do leste brasileiro. Acta Biologica Leopoldensia, 16: 51-80.

Buckup, P. A. 2011. The Eastern Brazilian Shield. Pp. 203-209. In: Albert, J. S. \& R. E. Reis (Eds.). Historical Biogeography of Neotropical Freshwater Fishes. Berkeley, University of California Press.

Carvalho, T. P. 2007. Distributional patterns of freshwater fishes in coastal Atlantic drainages of eastern Brazil: A preliminary study applying parsimony analysis of endemism. Darwiniana, 45: 65-67.

Costa, W. J. E. M. 1996. Phylogenetic and biogeographic analysis of the Neotropical annual fish genus Simpsonichthys (Cyprinodontiformes: Rivulidae). Journal of Comparative Biology, 1: 129-140.

Costa, W. J. E. M. 2001. The Neotropical annual fish genus Cynolebias (Cyprinodontiformes: Rivulidae): phylogenetic relationships, taxonomic revision and biogeography. Ichthyological Exploration of Freshwaters, 12: 333-383.

Costa, W. J. E. M. 2003. The Simpsonichthys flavicaudatus species group (Cyprinodontiformes: Rivulidae: Cynolebiatinae): phylogenetic relationships, taxonomic revision and biogeography. Ichthyological Exploration of Freshwaters, 14: 31-60.

Cracraft, J. 1991. Patterns of diversification within continental biotas: hierarchical congruence among the areas of endemism of Australian vertebrates. Australian Systematic Botany, 4: 211-227.

Eschmeyer, W. N. \& J. D. Fong. 2014. Species by Family/ Subfamily. Available from: http://research.calacademy.org/ research/ichthyology/catalog/SpeciesByFamily.asp. (02 Apr 2014).

Ferreira, K. M., N. A. Menezes \& I. Quagio-Grassioto. 2011. A new genus and two new species of Stevardiinae (Characiformes: Characidae) with a hypothesis on their relationships based on morphological and histological data. Neotropical Ichthyology, 9: 281-298.

Géry, J. 1969. The freshwater fishes of South America. Pp. 828848. In: Fitkau, E. J., J. Illies, H. Klinge, G. Shwabe \& H. Sioli (Eds.). Biogeography and Ecology in South America. The Hague, W. Junk. 
Goloboff, P. A., J. S. Farris \& K. C. Nixon. 2008. TNT, a free program for phylogenetic analysis. Cladistics, 24: 1-13.

Hales, J. \& P. Petry. 2013. Northeastern Mata Atlantica ecoregion. Avalaible from: http://www.feow.org/ecoregions/details/ northeastern_mata_atlantica (05 Dec 2013).

Haseman, J. D. 1912. Some factors of geographical distribution in South America. Annals of the New York Academy of Sciences, 22: 9-112.

Hennig, W. 1966. Phylogenetic Systematics. Ohio, Urbana: University of Illinois Press.

Jacobina, U. P., P. R. A. M. Affonso, P. L. S. Carneiro \& J. A. Dergam. 2009. Biogeography and comparative cytogenetics between two populations of Hoplias malabaricus (Bloch, 1794) (Ostariophysi: Erythrinidae) from coastal basins in the State of Bahia, Brazil. Neotropical Ichthyology, 7: 617-622.

Lima, F. C. T. \& A. C. Ribeiro. 2011. Continental-Scale Tectonic Controls of Biogeography and Ecology. Pp. 145-164. In: Albert, J. S. \& R. E. Reis (Eds.). Historical Biogeography of Neotropical Freshwater Fishes. Berkeley, University of California Press.

Lundberg, J. G., L. G. Marshall, J. Guerrero, B. Horton, M. C. S. L. Malabarba \& F. Wesselingh. 1998. The stage for Neotropical fish diversification: A history of tropical South American rivers. Pp. 13-48. In: Malabarba, L. R., R. E. Reis, R. P. Vari, Z. M. S. Lucena \& C. A. S. Lucena (Eds.). Phylogeny and Classification of Neotropical Fishes. Porto Alegre, Edipucrs.

Maddison, W. P. \& D. R. Maddison. 2009. Mesquite: a modular system for evolutionary analysis, version 2.72. Available from: http://mesquiteproject.org.

Malabarba, L. R., F. C. T. Lima \& S. H. Weitzman. 2004. A new species of Kolpotocheirodon (Teleostei: Characidae: Cheirodontinae: Compsurini) from Bahia, northeatern Brazil, with a new diagnosis of the genus. Proceedings of the Biological Society of Washington, 117: 317-329.

Mariangeles, A. H., R. E. Reis, A. J. Geneva \& M. H. Sabaj Pérez. 2013. Molecular phylogeny of thorny catfishes (Siluriformes: Doradidae). Molecular Phylogenetics and Evolution, 67: 560577.

Menezes, N. A. 1972. Distribuição e origem da fauna de peixes de água doce das grandes bacias fluviais do Brasil. Pp. 73-108. In: Poluição e Piscicultura. São Paulo, Faculdade de Saúde Pública da Universidade de São Paulo e Instituto de Pesca.

Menezes, N. A. 1988. Implications of the distribution patterns of the species of Oligosarcus (Teleostei, Characidae) from central and southern South America. Pp. 295-304. In: Vanzolini, P. E. \& W. R. Heyer (Eds.). Proceedings of a Workshop on Neotropical Distribution Patterns. Rio de Janeiro, Academia Brasileira de Ciências.

Menezes, N. A., A. C. Ribeiro, S. Weitzman \& R. A.Torres. 2008. Biogeography of Glandulocaudinae (Teleostei: Characiformes: Characidae) revisited: phylogenetic patterns, historical geology and genetic connectivity. Zootaxa, 1726: 33-38.

Montoya-Burgos, J. I. 2003. Historical biogeography of the catfish genus Hypostomus (Siluriformes: Loricariidae), with implications on the diversification of Neotropical ichthyofauna. Molecular Ecology, 12: 1855-1867.

Potter, P. E. 1997. The Mesozoic and Cenozoic paleodrainage of South America: A natural history. Journal of South American Earth Sciences, 10: 331-344.

Quevedo, R. \& R. E. Reis. 2002. Pogonopoma obscurum: a new species of loricariid catfish (Siluriformes: Loricariidae) from southern Brazil, with comments on the genus Pogonopoma. Copeia, 2002: 402-410.
Reis, R. E., E. H. L. Pereira \& J. W. Armbruster. 2006. Delturinae, a new loricariid catfish subfamily (Teleostei, Siluriformes), with revisions of Delturus and Hemipsilichthys. Zoological Journal of the Linnean Society, 147: 277-299.

Ribeiro, A. C. 2006. Tectonic history and the biogeography of the freshwater fishes from the coastal drainages of eastern Brazil: an example of faunal evolution associated with a divergent continental margin. Neotropical Ichthyology, 4: 225-246.

Rosa, R. S., N. A. Menezes, H. A. Britski, W. J. E. M. Costa \& F. Groth. 2004. Diversidade, padrões de distribuição e conservação dos peixes da Caatinga. Pp. 135-180. In: Leal, I. R., M. Tabarelli \& J. M. C. da Silva (Eds.). Ecologia e conservação da Caatinga. Recife, EDUFPE.

Rosen, B. R. 1988. From fossils to earth history: applied historical biogeography. Pp. 437-481. In: Myers, A. A. \& P. S. Giller (Eds.). Analytical biogeography, an integrated approach to the study of animal and plant distributions. London, Chapman and Hall.

Rosen, B. R. 1992. Empiricism and the biogeographical black box: concepts and methods in marine palaeobiogeography. Palaeogeography, Palaeoclimatology, Palaeoecology, 92: 171205.

Rosen, B. R. \& A. B. Smith. 1988. Tectonics from fossils? Analysis of reef coral and sea urchin distributions from late Cretaceous to Recent, using a new method. Pp. 275-306. In: AudleyCharles, M. G. \& A. Hallam (Eds.). Geological Society Special Publications, 37: Gondwana and Tethys. Oxford, Oxford University Press.

Saadi, A. 1998. A geomorfologia da Serra do Espinhaço em Minas Gerais e de suas margens. Geonomos, 3: 41-63.

Sarmento-Soares, L. M. \& R. F. Martins-Pinheiro. 2009. A fauna de peixes na bacia do rio dos Frades e microbacias vizinhas, extremo sul da Bahia, Brasil. Boletim do Museu de Biologia Mello Leitão, 26: 25-46.

Sarmento-Soares, L. M. \& R. F. Martins-Pinheiro. 2012. A fauna de peixes nas bacias do norte do Espírito Santo, Brasil. Sitientibus série Ciências Biológicas 12: 27-52.

Sarmento-Soares, L. M., R. Mazzoni \& R. F. Martins-Pinheiro. 2007. A fauna de peixes na bacia do Rio Peruípe, extremo Sul da Bahia. Biota Neotropica, 7: 291-308.

Sarmento-Soares, L. M., R. Mazzoni \& R. F. Martins-Pinheiro. 2008. A fauna de peixes dos Rios dos Portos Seguros, extremo sul da Bahia, Brasil. Boletim do Museu de Biologia Mello Leitão, 24: 119-142.

Sarmento-Soares, L. M., R. Mazzoni \& R. F. Martins-Pinheiro. 2009a. A fauna de peixes nas bacias litorâneas da Costa do Descobrimento, extremo sul da Bahia, Brasil. Sitientibus Série Ciências Biológicas, 9: 139-157.

Sarmento-Soares, L. M., R. Mazzoni \& R. F. Martins-Pinheiro. 2009b. A fauna de peixes na bacia do Rio Jucuruçu, leste de Minas Gerais e extremo Sul da Bahia. Pan-American Journal of Aquatic Sciences, 4: 193-207.

Schaefer, S. A. 1997. The Neotropical cascudinhos: systematics and biogeography of the Otocinclus catfishes (Siluriformes: Loricariidae). Proceedings of the Academy of Natural Sciences of Philadelphia, 148: 1-120.

Serra, J. P., F. R. Carvalho \& F. Langeani. 2007. Ichthyofauna of the rio Itatinga in the Parque das Neblinas, Bertioga, São Paulo State: composition and biogeography. Biota Neotropica, 7: 8186.

Stiassny, M. \& M. C. C. de Pinna. 1994. Basal taxa and the role of cladistic patterns in the evaluation of conservation priorities: 
A view from freshwater. Pp. 235-249. In: Forey, P. L., C. J. Humphries \& R. I. Vane-Wright (Eds.). Systematics and conservation evaluation. The Systematics Association Special. Oxford, Oxford (United Kingdom) Clarendon Press.

Vari, R. P. 1988. The Curimatidae, a lowland Neotropical fish family (Pisces: Characiformes): distribution, endemism, and phylogenetic biogeography. Pp. 343-277. In: Heyer, W. R. \& P. E. Vanzolini (Eds.). Proceedings of a workshop on Neotropical Distribution Patterns. Rio de Janeiro, Academia Brasileira de Ciências.

Vari, R. P. \& S. H. Weitzman. 1990. A review of the phylogenetic biogeography of the freshwater fishes of South America. Pp. 381-393. In: Peters, G. \& R. Hutterer (Eds.). Vertebrates in the tropics: Proceedings of the International Symposium on Vertebrate Biogeography and Systematics in the Tropics. Bonn, Museum Alexander Koenig.
Weitzman, S. H., N. A. Menezes \& M. J. Weitzman. 1988. Phylogenetic biogeography of the glandulocaudini (Teleostei: Characiformes, Characidae) with comments on the distribution of other freshwater fishes in eastern and southeastern Brazil. Pp. 379-427. In: Heyer, W. R. \& P. E. Vanzolini (Eds.). Proceedings of a workshop on Neotropical Distribution Patterns. Rio de Janeiro, Academia Brasileira de Ciências.

Zanata, A. M. \& J. P. Serra. 2010. Hasemania piatan, a new characid species (Characiformes: Characidae) from headwaters of rio de Contas, Bahia, Brazil. Neotropical Ichthyology, 8: 21-26.

Submitted December 16, 2013

Accepted July 3, 2014 by Luiz Rocha Published December 27, 2014 